\title{
A highly emissive distyrylthieno[3,2-b]thiophene based red luminescent organic single crystal: Aggregation induced emission, Optical waveguide edge emission, and Balanced ambipolar carrier transport
}

Shuai Mu, ${ }^{a}$ Kazuaki Oniwa, ${ }^{\mathrm{b}}$ Tienan Jin, ${ }^{\mathrm{b}}$ Naoki Asao, ${ }^{\mathrm{b}}$ Masahiro Yamashita, ${ }^{\mathrm{a}}$ Shinya Takaishi ${ }^{\mathrm{a} *}$

${ }^{a}$ Department of Chemistry, Graduate School of Science, Tohoku University, 6-3 Aramaki-Aza-Aoba, Aoba-ku, Sendai, Miyagi, 980-8578, Japan.

${ }^{\mathrm{b}}$ WPI-Advanced Institute for Materials Research, Tohoku University, Sendai 980-8577, Japan

*Corresponding author. E-mail: takaishi@mail.tains.tohoku.ac.jp; Fax: +81-22-795-6547.

\begin{abstract}
A highly emissive red luminescent single crystal which shows aggregation induced emission (AIE) property and optical waveguide edge emission based on small organic functional molecule, cyano-substituted 2,5-di((E)-styryl)thieno[3,2-b]thiophene (CNP2V2TT) has been prepared by the physical vapor transport (PVT) method. The fluorescence quantum efficiency of crystal is up to $37 \%$ and an emission peak maximum $\left(\lambda_{\max }\right)$ locates at $645 \mathrm{~nm}$. Cystallographic data indicate that uniaxially oriented molecular packing with slipped face-to-face $\pi-\pi$ stacking forms by the hydrogen bonding network among CNP2V2TT molecules. The single crystal FET devices were fabricated using Au and $\mathrm{Ca}$ as hole and electron injection electrodes, respectively. The molecular design, introducing cyano groups into molecular skeleton, effectively lower the LUMO level and achieve well-balanced ambipolar electron $\left(0.13 \mathrm{~cm}^{2} \mathrm{~V}^{-1} \mathrm{~s}^{-1}\right)$ and hole $\left(0.085 \mathrm{~cm}^{2} \mathrm{~V}^{-1} \mathrm{~s}^{-1}\right)$ mobilities.
\end{abstract}




\section{Graphical abstract}

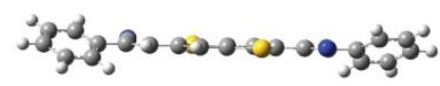

Twisted conformation

PL yield $<1 \%$

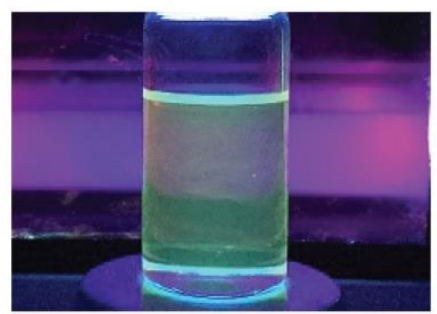

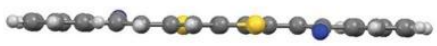

Coplanar conformation

PL yield up to $37 \%$

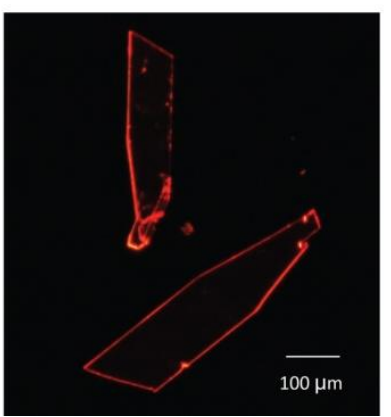

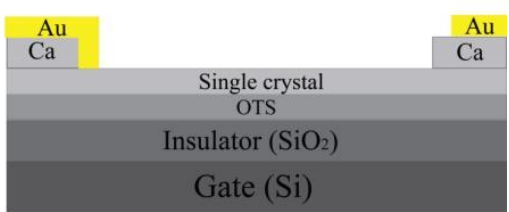

$\mu_{\mathrm{e}}=0.13 \mathrm{~cm}^{2} / \mathrm{Vs}$

$\mu_{\mathrm{h}}=0.085 \mathrm{~cm}^{2} / \mathrm{Vs}$

\section{Keywords}

Red luminescence; Highly emissive crystal; Aggregation induced emission (AIE); Optical waveguide edge emission; Cyano-substituted; Balanced ambipolar carrier transport

\section{Introduction}

Development of highly emissive materials in the solid state is a crucial prerequisite for optoelectronic and photonic devices and has become a hot topic of scientific research in the past decades [1-3]. In RGB (red, green, blue) luminescence, photoluminescence (PL) quantum yield of red color is normally much lower than that of blue and green colors. Red luminescence comes from the luminophor with a narrow HOMO-LUMO energy gap, which is easy to result in non-radiative recombination of excitons. On the other hand, significant aggregation-caused quenching (ACQ) effect also renders the red luminophor weakly emissive. An opposite effect named aggregation induced emission (AIE) was found by Tang group [4]. AIE effect originates from the restriction of bond rotation in the aggregation state, which can reduce the nonradiative loss and result in the luminescence enhancement $[5,6]$. AIE molecules possess unusual high luminescence in the solid state but hardly emissive in the solution 
state, which gives a promising strategy to design highly emissive molecular solid for optoelectronic and photonic applications.

Recently, the single crystal (SC) of linear $\pi$-conjugated organic molecule attracts much attention due to its distinct merits such as few grain boundaries and defects which contribute to achieve good charge transport performance $[7,8]$. In addition, some single crystals based on linear $\pi$-conjugated organic molecule exhibit optical waveguide edge emission, which is determined by the well-defined transition dipole alignment in the crystal [9-12]. Unlike amorphous materials, organic single crystals exhibit various regular intermolecular aggregation manners such as $\mathrm{H}$-aggregate or J-aggregate. It is well known that organic crystal exhibits distinctive shift in absorption spectrum and emission spectrum depending on the aggregation manner which characterizing transition dipole alignment in the crystal [13-16]. Therefore, small change in aggregation manner can induce large difference in optical properties of organic single crystal, which gives a unique horizon to modulate optical properties of organic single crystal by careful design of molecular features. An effective route to modulate aggregation manner is to introduce cyano groups into molecular skeleton. Cyano groups can form $\mathrm{C}-\mathrm{H} \cdots \mathrm{N}$ hydrogen bonding which can effectively change the typical herringbone stacking motif of organic single crystal. To date, some researches introduce cyano-substitution into the vinylene unit or the terminal phenyls of molecular skeleton, giving rise to different aggregation manners, have been reported [17-20]. To the best of our knowledge, highly emissive red-emitting materials with confined emitting properties for optoelectronic devices are very limited.

In this work, we report on synthesis, crystal structure and physical properties in a novel molecular crystal of CNP2V2TT. CNP2V2TT single crystal exhibits high quality red luminescence and aggregation induced emission (AIE) property with a very high photoluminescence quantum yield up to 
37\%. CNP2V2TT single crystal also exhibits optical waveguide edge emission. By introducing cyano groups into the molecular skeleton, the strong hydrogen bonding network forms and plays a crucial role in the molecular packing and optical properties of CNP2V2TT crystal. On the other hand, cyano group is a strong electron withdrawing group, which can significantly lower LUMO level and improve the electron injection performance. We fabricated single crystal field effect transistor (SC-FET) devices using $\mathrm{Au}$ and $\mathrm{Ca}$ as hole and electron injection electrodes and balanced ambipolar carrier transport was achieved.

\section{Experimantal section}

\subsection{Synthesis}

The synthetic route of CNP2V2TT molecule is shown as scheme 1. To a solution of thieno[3, 2-b]thiophene $1(0.56 \mathrm{~g}, 4 \mathrm{mmol})$ and in dry $\mathrm{Et}_{2} \mathrm{O} 50 \mathrm{~mL}$, n-butyl lithium in n-hexane $(6 \mathrm{~mL}, 10 \mathrm{mmol}$, $1.6 \mathrm{M}$ ) was added dropwise under an argon atmosphere at $0{ }^{\circ} \mathrm{C}$. The stirring was continued for $2 \mathrm{~h}$ and subsequently DMF ( $1 \mathrm{ml}, 13 \mathrm{mmol})$ was added and stirred for additional 1 day at ambient temperature. The solution was quenched with diluted aqueous $\mathrm{HCl}$ under vigorous stirring. The precipitate was filtered and dried to give a yellow solid 2 (354 mg, $1.8 \mathrm{mmol}$, yield 45\%), which was practically pure and can be used to the next reaction. $1 \mathrm{H} \mathrm{NMR}(500 \mathrm{MHz}, \mathrm{CDCl} 3) \delta 8.01(\mathrm{~s}, 2 \mathrm{H}, \mathrm{Ar}-\mathrm{H})$, 10.05 (s, 2H, CHO); CNP2V2TT was synthesized via Knoevenagel condensation. To a solution of compound $2(100 \mathrm{mg}, 0.5 \mathrm{mmol})$ and benzylcyanide $(113 \mathrm{mg}, 1.1 \mathrm{mmol})$ in $20 \mathrm{ml} \mathrm{THF}$, a solvent of potassium tert-butoxide $(1 \mathrm{mg} / \mathrm{ml})$ in $20 \mathrm{ml}$ tert-butoxide was added dropwise within $5 \mathrm{~min}$, then 4 drops $(\sim 0.2 \mathrm{ml})$ of tetra-n-butylammonium hydroxide $(1 \mathrm{M}$ in methanol) were added. The mixture stirred at ambient temperature for $10 \mathrm{~h}$. The orange precipitate was collected by filtration and washed with methanol. The material was further purified by three sublimations to afford CNP2V2TT as a red 
solid (36 mg, $0.076 \mathrm{mmol}$, yield 18\%). CNP2V2TT exhibits poor solubility in commonly used organic solvent. Anal. Calcd for $\mathrm{C}_{24} \mathrm{H}_{14} \mathrm{~N}_{2} \mathrm{~S}_{2}$ : C, 73.07; H, 3.58. N, 7.10; Found: C, 72.94; H 3.91, N 7.08.

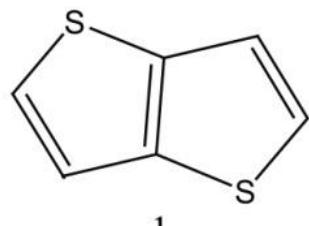

1

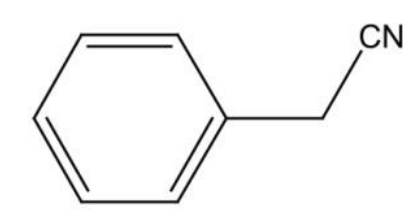

$\mathrm{TBAH} / \mathrm{t}-\mathrm{BuOK}$

$\mathrm{THF} / \mathrm{t}-\mathrm{BuOH}$

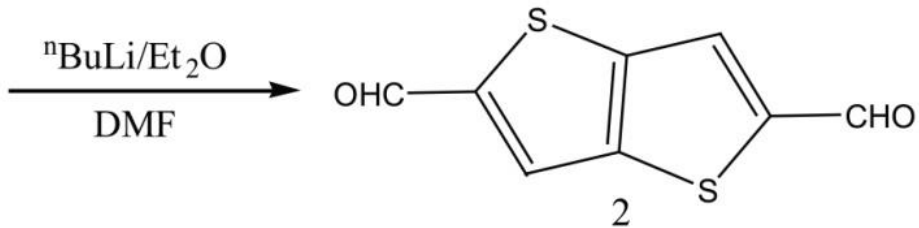

2

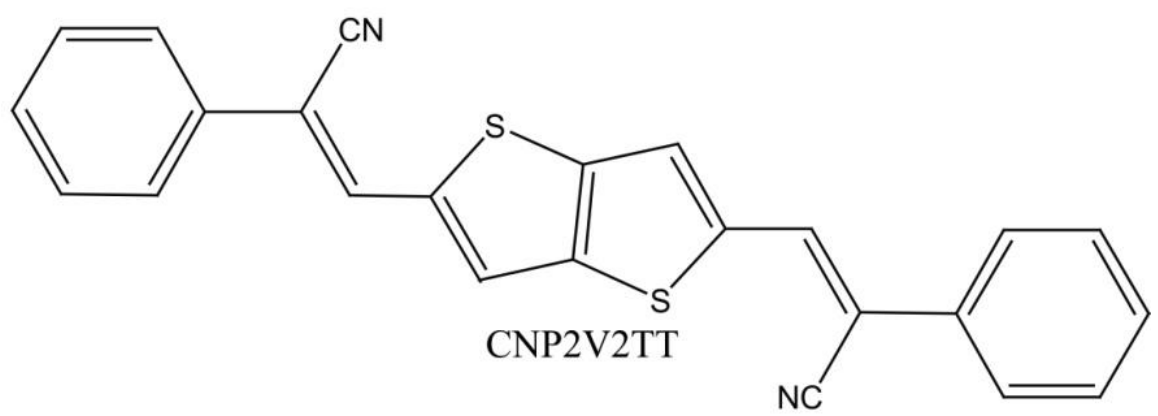

Scheme. 1 Synthetic route of CNP2V2TT.

\subsection{Crystal growth}

Thin platelet crystals of CNP2V2TT were grown by physical vapor transport (PVT) method. Ultrapure argon was used as the carrier gas. TG/DTA measurement exhibits high heat stability of CNP2V2TT crystal over $350{ }^{\circ} \mathrm{C}$ (see Fig. S1 in the supporting data). To grow high quality crystals, different temperature zones were used. The high temperature zone $\left(330{ }^{\circ} \mathrm{C}\right)$ was used as the sublimating region, while the low temperature zone $\left(250^{\circ} \mathrm{C}\right)$ was used for crystal growth.

\subsection{Instrumental process}

Optical absorption spectrum in diluted solution was measured using Shimadzu UV-3100 spectrometer.

We obtained absorption spectrum of CNP2V2TT crystal by transforming diffuse reflection spectrum of CNP2V2TT microcrystal doped $\mathrm{BaSO}_{4}$ film measured on the Shimadzu UV-3100 spectrometer coupled with an integrating sphere. In details, $\mathrm{BaSO}_{4}$ was used as $100 \%$ reflection standard, which was filled into the sample cell and compressed. Then several milligrams of platelet CNP2V2TT 
crystals obtained by PVT method were carefully ground with a suitable amount of $\mathrm{BaSO}_{4}$ in order to make CNP2V2TT microcrystals homogeneously distributed in $\mathrm{BaSO}_{4}$ and compressed into a thin film on the $\mathrm{BaSO}_{4}$ substrate. By using integrating sphere, we measured diffuse reflection spectrum of the sample and then transformed it into absorption spectrum. Fluorescence spectra in the diluted state were measured on a JASCO FP-8300 spectrophotometer. Fluorescence spectra in the solid state and absolute quantum efficiencies were measured by a photon-counting method using an integration sphere (C9920-2, Hamamatsu Co., Japan). Single crystals were grown by a physical vapor transport (PVT) method under argon gas atmosphere using a separation temperature controller (AMF-9P-III, ASAHI RIKA). Single-crystal crystallographic data were collected on a Rigaku Saturn70 CCD diffractometer with graphite-monochromated Mo K $\alpha$ radiation $(\lambda=0.71075 \AA)$ produced using a VariMax micro-focus X-ray rotating anode source at $93 \mathrm{~K}$. PXRD measurements were performed at room $\mathrm{T}$ in air on a BRUKER D2 PHASER. Elemental analysis was measured on J-SCIENCE Lab JM-10 and YANAKOHNS-ah/HSU-20 in Research and Analytical Center for Giant Molecules, Tohoku University. The TGA analyses were performed on a SHIMADZU DTG-60H instrument at $10{ }^{\circ} \mathrm{C} \min ^{-1}$ under a nitrogen atmosphere. The FET transport characteristics were carried out inside a $\mathrm{N}_{2}$-filled glove box by using an Agilent Technology B1500A semiconductor parameter analyzer.

\section{Result and discussion}

\subsection{Optical properties}

Optical properties of CNP2V2TT were characterized in solution and solid states, respectively (see Fig. 2). The absorption peak of CNP2V2TT molecule in the solution locates at $423 \mathrm{~nm}$. The absorption peak of CNP2V2TT microcrystals doped $\mathrm{BaSO}_{4}$ film exhibits an obvious blue shift, which locates at 
$400 \mathrm{~nm}$. CNP2V2TT exhibits single molecule state in the diluted solution while H-aggregate in the CNP2V2TT microcrystal doped $\mathrm{BaSO}_{4}$ film based on single crystal structure. According to excitonic coupling in dimers, in H-aggregate case, the lower one of two excited states to the ground state is optically transition forbidden, which means more excitation energy is demanded for the absorption of crystal against single molecule. (see inset of Fig. 2). In order to show that the differences in the absorption spectra in the crystalline state and solution state is not due to the difference in the molecular conformation but due to the difference in the aggregation manners, we calculated UV-Vis spectra of CNP2V2TT single molecule in the optimized conformation (= diluted solution) and conformation in the single crystal, respectively, by TD-DFT method (see Fig. S3 and Fig. S4 in supporting data). From calculated results, we can find CNP2V2TT molecule exhibits almost same excitation energy in the two conformations, indicating that the difference in the absorption spectra mainly originates from the different aggregation manners of CNP2V2TT molecule in the diluted solution (single molecule state) and single crystal (H-aggregate). Emission spectrum of CNP2V2TT in solution is accompanied with vibronic structure and two clear emission peaks at $491 \mathrm{~nm}$ and $528 \mathrm{~nm}$ can be observed. However, emission spectrum of CNP2V2TT crystal shows unstructured and emission peak locates at $645 \mathrm{~nm}$. Compared with the highest emission peak in the solution state $(491 \mathrm{~nm})$, emission peak of CNP2V2TT crystal exhibits a strong bathochromic (red) shift over $0.6 \mathrm{eV}$ against solution, which is obviously larger by comparison with that in typical herringbone like aggregate. Clear red edge emission can be observed from photoluminescent image of CNP2V2TT crystal under $365 \mathrm{~nm} \mathrm{UV} \mathrm{light} \mathrm{(see} \mathrm{Fig.} \mathrm{1(c)).}$ In solution, CNP2V2TT shows a very low PL quantum yield less than $1 \%$. In contrast, CNP2V2TT crystal exhibits strong aggregation-induced emission (AIE) with a very high photoluminescence (PL) quantum yield up to $37 \%$. This photoluminescence enhancement is similar with other twisted 
conjugated molecules, such as CNDPDSB [21], CNDSB [22], which show very low luminescence in solution but strongly enhanced emission in the solid state. In the diluted state, CNP2V2TT molecule exhibits a twisted conformation (see Fig 1(a)). In the single crystal, the tight intermolecular packing in the solid state restricts the bond rotation and gives rise to nearly planar molecular conformation (see Fig. 1(b)), which plays a crucial role to the aggregation induced emission (AIE) behavior.

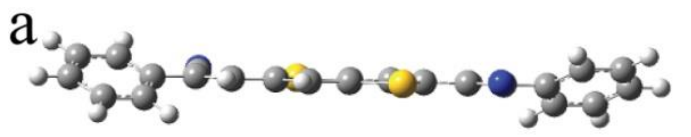

Twisted conformation

PL yield $<1 \%$

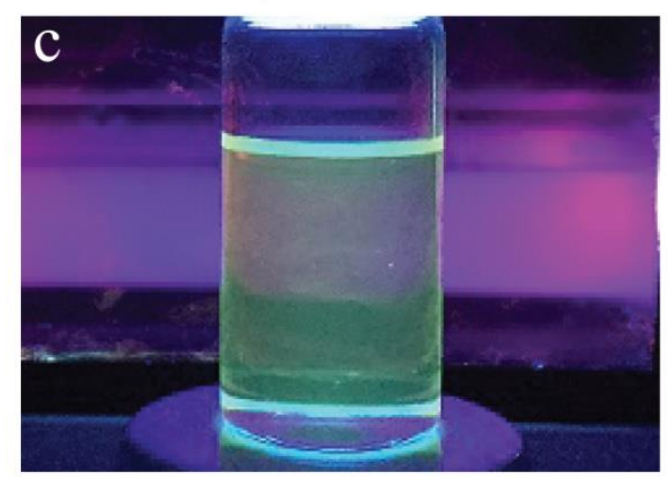

b

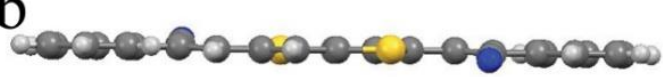

Coplanar conformation

PL yield up to $37 \%$

AIE

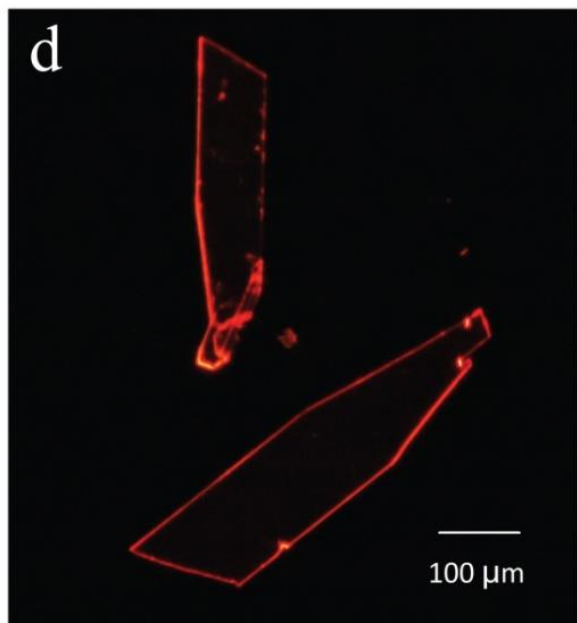

Fig. 1 Molecular conformation in the diluted solution calculated by the Gaussian 09 packge [25] (a) and very weak luminescence from diluted chloroform under $365 \mathrm{~nm}$ UV lamp (c). Molecular conformation in the single crystal (b) and clear edge emission from the single crystal (d). 


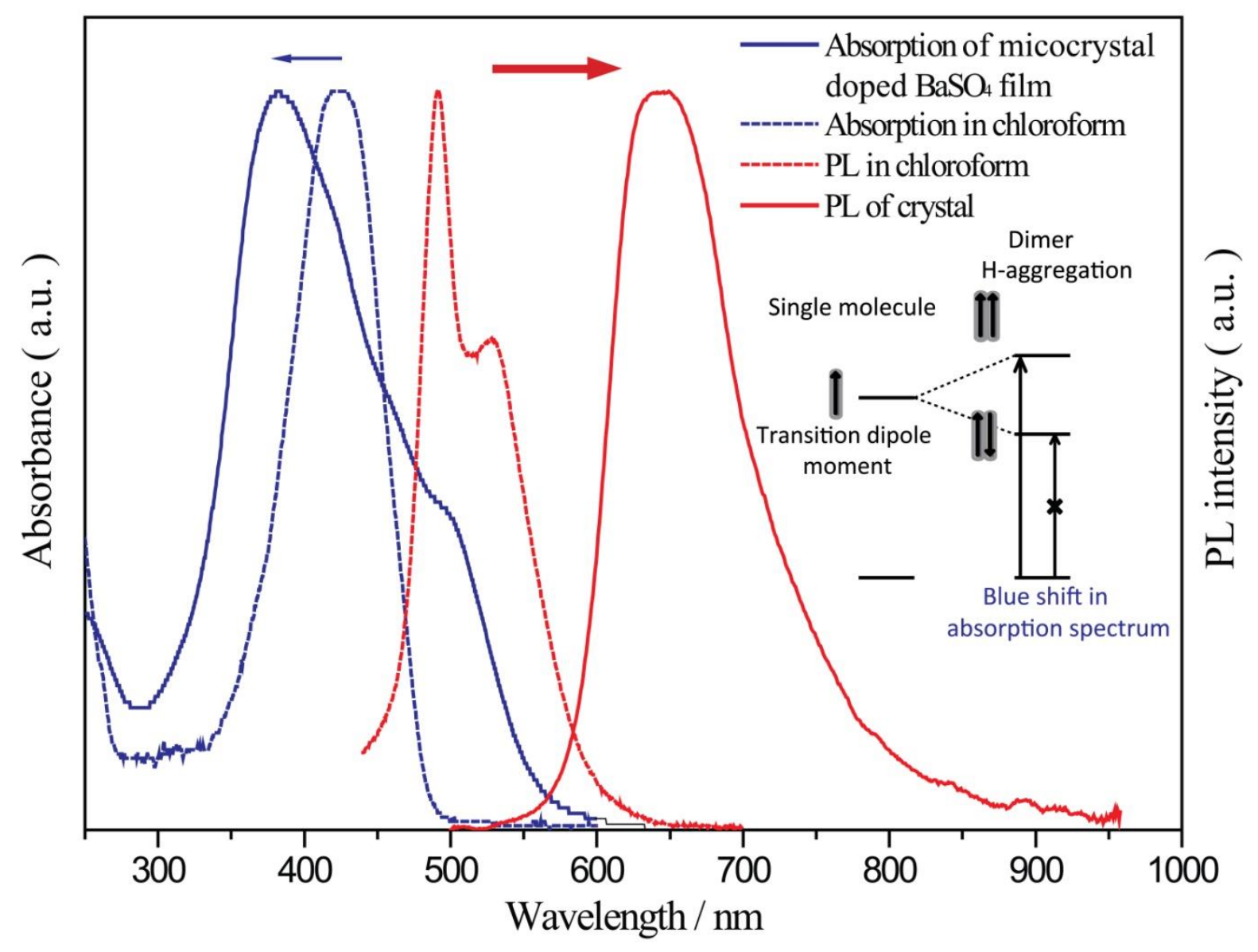

Fig. 2 Normalized absorption and emission spectra of CNP2V2TT in chloroform (dashed line) and solid state (solid line). Inset: schematic diagram of excitonic coupling in H-aggregate.

\subsection{Structure analysis}

For a better understanding of optical properties, single crystal structure of CNP2V2TT was measured. CNP2V2TT crystal shows triclinic lattice with P-1 space group. Cell parameters are: $a=3.832(5) \AA, \quad b=5.947(6) \AA, c=20.569(19) \AA, \alpha=76.44(5)^{\circ}, \beta=84.88(5)^{\circ}, \gamma=$ 84.96(4) $)^{\circ}$ and $Z=1$. CNP2V2TT exhibits nearly planar molecular conformation and. molecules stack into a slipped column by efficient face to face $\pi-\pi$ interaction with a short inter-plane distance of $3.47 \AA$ (see Fig. 3(c)). As cyano groups are introduced, strong N...H-C hydrogen bonds (red dotted lines) forms between the cyano group and two hydrogen atoms 
from the vinylene unit and the terminal phenyls of the neighboring molecule, resulting in uniaxially oriented molecular packing. Fig 3(a) shows CNP2V2TT crystal image under visible light. From the inset of Fig 1(d) and Fig 3(a), we can find crystal growth exhibits a tendency to form rhomboid shape, which matches well with the perspective view of molecular packing in the CNP2V2TT crystal from the top ab-plane (see Fig 2(b)). The powder X-ray diffraction (PXRD) pattern of the solid samples is shown in Fig. S2 of supporting data.

Here we consider the origin of large Stokes shift and edge emission from the structural feature of CNP2V2TT crystal. The angle between the molecular long axis of CNP2V2TT and ab-plane is $75.2^{\circ}$ (see Fig. 3(d)). Based on TD-DFT calculation, the transition dipole moment of CNP2V2TT molecule is identified to be nearly parallel to the long axis of the molecule [22-24]. Thus it is reasonable that the angle $\theta$ between the transition dipole moment of CNP2V2TT molecule and crystal ab-plane is ca. $75.2^{\circ}$, which indicates the transition dipole moments are nearly perpendicular to the crystal ab plane. Schematic representation of traveling direction of light is illustrated in the inset of Fig. 3(d). Waveguide behavior in the organic single crystal is determined by transition dipole alignment in the crystal. Because traveling direction of light is perpendicular to direction of transition dipole moment, by considering Snell's law and typical refractive index of organic crystals $(n=1.5)$, as a result critical angle $\left(\theta_{c}\right)$ at the crystal/air interface is ca. $41.8^{\circ}$. Thus, this crystal meets the criteria for the total reflection because $\alpha>\theta_{c}$, thereby limiting photons emitted from the edge of the crystal. According to the simplified Kasha point-dipole model for excitonic coupling in dimmers $[14,16]$, it regards no excitonic splitting occurs when $\theta=54.7^{\circ}$ called 'magic angle', at which electrical dipole-dipole interaction becomes zero. So it is traditionally regarded that $\mathrm{H}$-aggregate forms when $\theta$ is 
larger than $54.7^{\circ}$ while $\mathrm{J}$-aggregate forms when $\theta$ is smaller than $54.7^{\circ}$. In the $\mathrm{CNP} 2 \mathrm{~V} 2 \mathrm{TT}$ crystal, the angle $\theta$ is much larger than $54.7^{\circ}$, which definitively induces efficient excitonic splitting, giving rise to H-type aggregate. Then considering unstructured emission spectrum which originates from the vibronic coupling between efficient intermolecular $\pi-\pi$ stacking [16], we can conclude that unusual large bathochromic (red) shift in emission from crystal against solution contributes to achieve high quality red luminescence, which is due to some degree of excimeric contributions although crystal exhibits some degree of slipped stack motif [26,27].
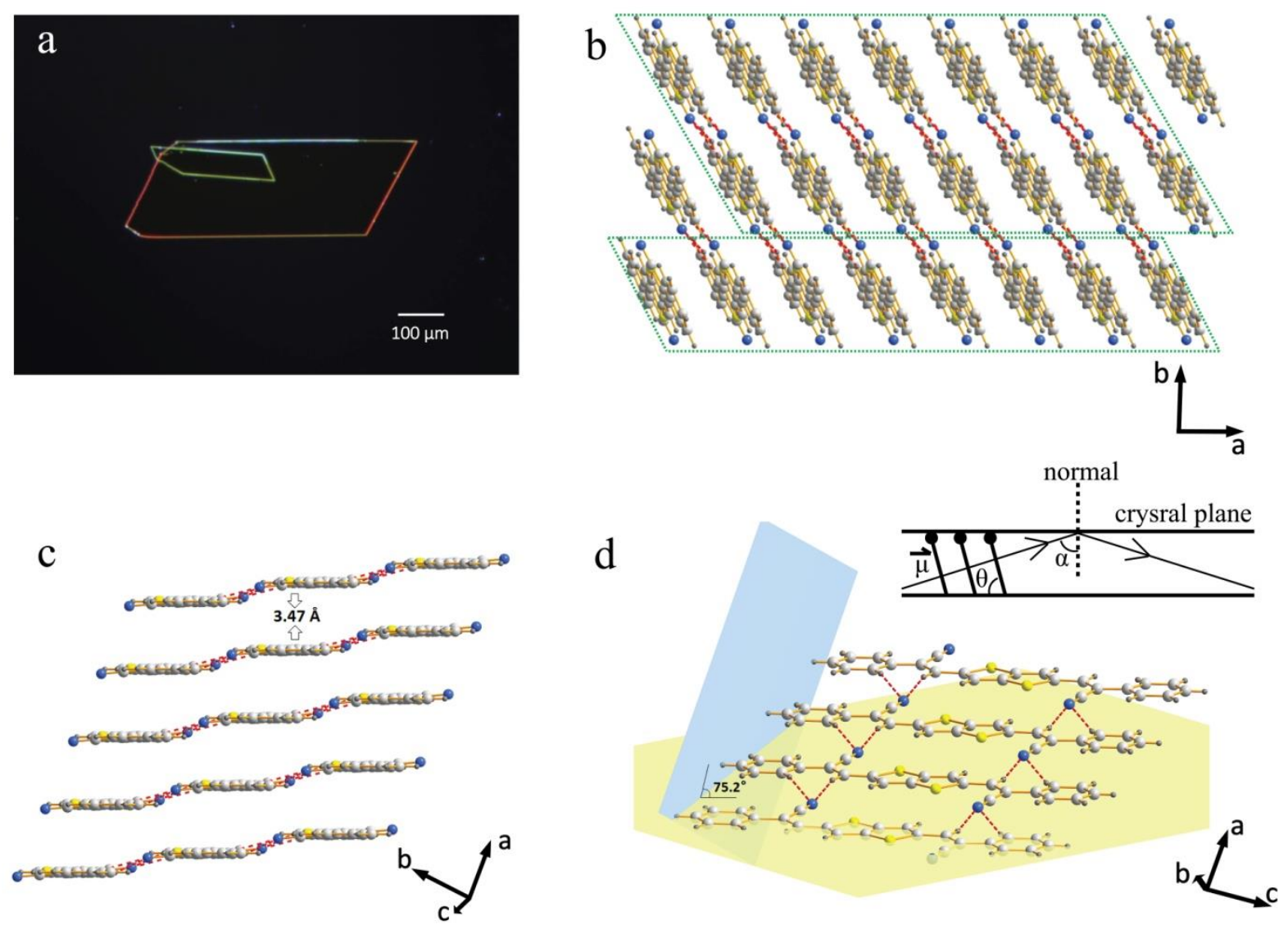

Fig. 3 CNP2V2TT crystal under visible light (a). Crystal structures of CNP2V2TT on the ab-plane (b).

Side view and the illustration of intermolecular $\pi-\pi$ stacking (c). Side view of intermolecular interactions (red dotted line represents the hydrogen bonding; blue plane represents the ab-plane; yellow plane represents the molecular plane of CNP2V2TT) (d). Inset: the illustration of transition dipole alignment 
and photons transport pathway in the crystal.

\subsection{SC-FET measurement}

In organic semiconductors, charge carriers transport along molecular $\pi$-orbitals. Thus overlap degree of molecular orbitals (electronic coupling) significantly impact charge transport of organic semiconductors. $\mathrm{H}$-aggregates with ideal $\pi$-stack exhibit efficient electronic coupling and thus it is reasonable that charge transport properties can be effectively improved along the molecular packing direction. For clarifying the charge transport properties of the CNP2V2TT crystal, we fabricated the single crystal FET devices (SC-FETs). The SC-FETs employ the top contact/bottom gate configuration (see Fig. 4(b)). For minimizing electron traps at the interface between the gate electrode and organic semiconductor, the octadecyltrichlorosilane (OTS) buffer layer was coated on the $\mathrm{SiO}_{2}$ surface by immersing the silicon wafer into the toluene solution containing OTS for two days at room temperature. A thin CNP2V2TT single crystal was laminated on the substrate by electrostatic adhesion. Au and $\mathrm{Ca}$ were employed as hole and electron injection electrodes with thickness of $50 \mathrm{~nm}$. FET measurements were conducted in the glovebox. As structure analysis, molecular packing is along the a-axis. Thus it is reasonable to make the conduction channel along the a-axis (between parallel orientation to the short edge and perpendicular orientation to the long edge of rhomboid-shape crystal, as shown in Fig. 4(c)). Comparing work function of $\mathrm{Au}(5.1 \mathrm{eV})$ and $\mathrm{Ca}(2.87 \mathrm{eV})$ with HOMO/LUMO level of CNP2V2TT calculated by the DFT method (see Fig 4(a)), the Schottky barrier for electron injection is slightly smaller than the hole injection. Fig 4(d) illustrates the transfer characteristics of CNP2V2TT single crystal device at various source-drain voltages $\left(\mathrm{V}_{\mathrm{ds}}\right)$. As predicted, typical V-shape transfer curves for ambipolar transport clearly indicate in P-channel 
and N-channel measurements, respectively. Output characteristics from same CNP2V2TT single crystal device is shown in Fig 4(e). Both electron and hole transport are evident in the $\mathrm{N}$-channel and P-channel measurements, respectively. Balanced ambipolar performance with electron mobility $\mu_{\mathrm{e}}$ up to $0.13 \mathrm{~cm}^{2} \mathrm{~V}^{-1} \mathrm{~s}^{-1}$ and hole mobility $\mu_{\mathrm{h}}$ up to $0.085 \mathrm{~cm}^{2} \mathrm{~V}^{-1} \mathrm{~s}^{-1}$ were achieved.
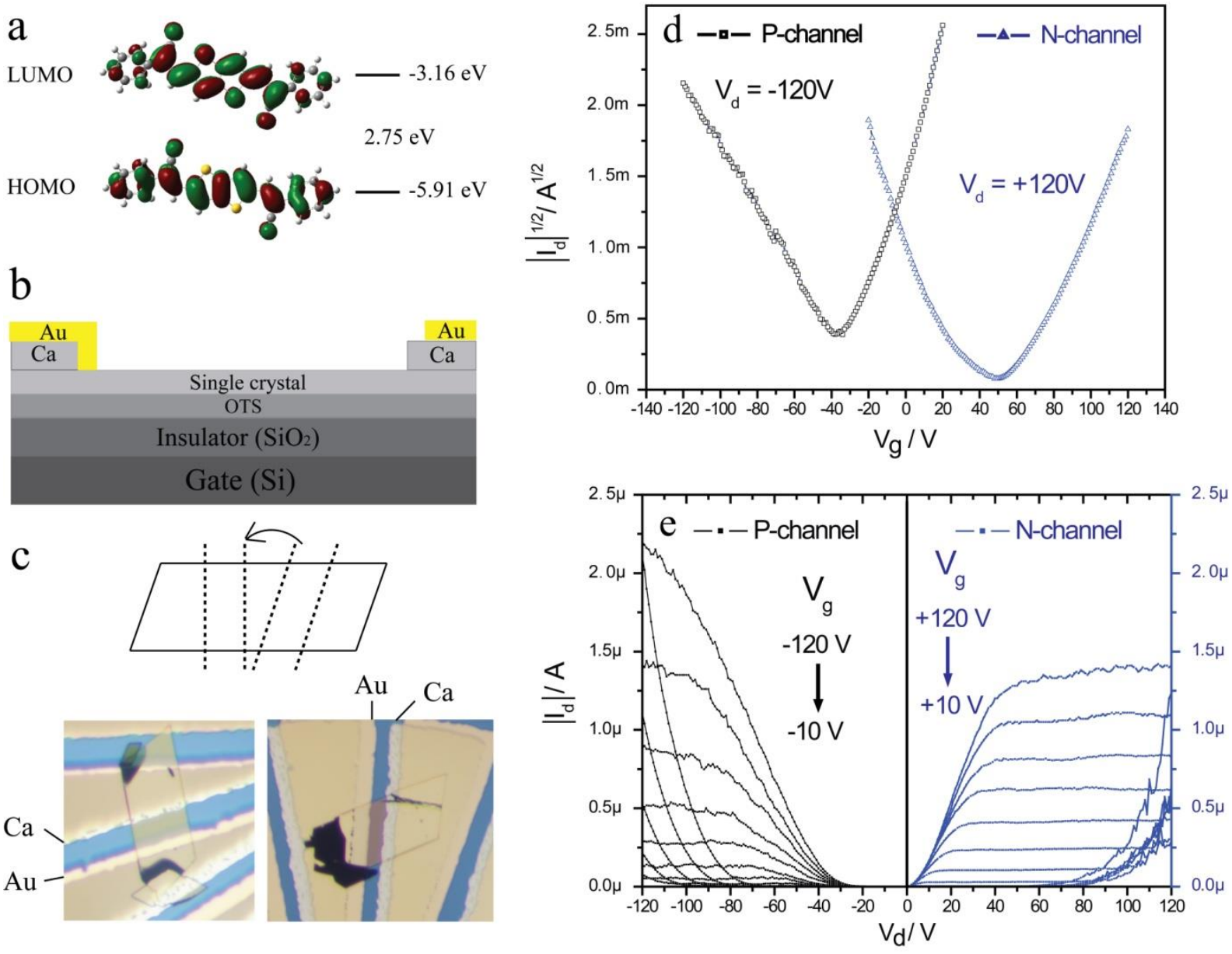

Fig 4. DFT-calculated (B3LYP/6-311G) molecular orbitals and HOMO/LUMO levels (a). Schematic representation of the SC-FET ( b). Optical photomicrographs of the SC-FET devices (c). Transfer (d) and output (e) characteristics of the SC-FET (Vg from -10 to $-120 \mathrm{~V}$ and from 10 to $120 \mathrm{~V}$ in steps of $10 \mathrm{~V}$. The channel width (W): $141 \mu \mathrm{m}$ and channel length (L): $30 \mu \mathrm{m}$ ).

\section{Conclusion}

In conclusion, we have developed a high quality red luminescent $\left(\lambda_{\max }=645 \mathrm{~nm}\right)$ organic single 
crystal (CNP2V2TT), which exhibits aggregation induced emission (AIE) property with a very high PL quantum yield up to 37\%. CNP2V2TT single crystal also exhibits optical waveguide edge emission. Cystallographic data indicates the existence of $\mathrm{C}-\mathrm{H} \cdots \mathrm{N}$ hydrogen bonding change the common herringbone stacking motif to an uniaxially oriented molecular packing with slipped face to face intermolecular $\pi$ - $\pi$ stacking. On the other hand, introduction of cyano group into molecular skeleton can effectively lower the LUMO level and improve the electron injection behavior. Balanced ambipolar charge mobilities with $\mu_{\mathrm{e}}$ up to $0.13 \mathrm{~cm}^{2} \mathrm{~V}^{-1} \mathrm{~s}^{-1}$ and $\mu_{\mathrm{h}}$ up to $0.085 \mathrm{~cm}^{2} \mathrm{~V}^{-1} \mathrm{~s}^{-1}$ were achieved from the single crystal FET devices using $\mathrm{Au}$ and $\mathrm{Ca}$ as hole and electron injection electrodes, respectively. Our research demonstrates CNP2V2TT sing crystal is a promising highly emissive red-emitting material with confined emitting properties for optoelectronic and photonic applications.

\section{Achnowlegement}

This work was partly supported by a JSPS KAKENHI grant (A) 26248015, a Grant-in-Aid for Young Scientist (B) 25810032, CREST, JST and Research Fellowships of Japan Society for the Promotion of Science for Young Scientists.

\section{References}

[1] Y. Hong, J.W.Y. Lam, B.Z. Tang, Chem. Soc. Rev. 40 (2011) 5361-5388.

[2] L. Basabe-Desmonts, D.N. Reinhoudt and M. Crego-Calama, Chem. Soc. Rev. 36 (2007) 993-1017.

[3] I.D.W. Samuel and G.A. Turnbull, Chem. Rev. 107 (2007), 1272-1295.

[4] J. Luo, Z. Xie, J.W.Y. Lam, L. Cheng, H. Chen, C. Qiu, H.S. Kwok, X. Zhan, Y. Liu, D. Zhu and B.Z. Tang, Chem. Commun. (2001) 1740-1741.

[5] B.-K. An, J. Gierschner, S.Y. Young, Acc. Chem. Res. 45 (2012) 544-554.

[6] H. Tong, Y.Q. Dong, M. Haussler, J.W.Y. Lam, H.H.Y. Sung, I.D. Williams, J.Z. Sun and B.Z. Tang, Chem. 
Commun. (2006) 1133-1135.

[7] S.Z. Bisri, T. Takenobu, Y. Yomogida, H. Shimotani, T. Yamao, S. Hotta and Y. Iwasa, Adv. Funct. Mater. 19 (2009) 1728-1735.

[8] T. Takenobu, S.Z. Bisri, T. Takahashi, M. Yahiro, C. Adachi and Y. Iwasa, Phys. Rev. Lett. 100 (2008) 066601.

[9] S.Z. Bisri, T. Takenobub and Y. Iwasa, J. Mater. Chem. C. 2 (2014) 2827-2836.

[10] H.-H. Fang, J. Yang, J. Feng, T. Yamao, S. Hotta and H.-B. Sun, Laser Photonics Rev. 8 (2014), 687-715.

[11] T. Komori, H. Nakanotani, T. Yasuda and C. Adachi, J. Mater. Chem. C. 2 (2014) 4918-4921.

[12] S. Hotta, T. Yamao, J. Mater. Chem. 21 (2011) 1295-1304.

[13] J. Cornil, D.A. Dos Santos, X. Crispin, R. Silbey and J.L. Bredas, J. Am. Chem. Soc. 120 (1998) 1289-1299.

[14] J. Cornil, D. Beljonne, J.-P. Calbert and J.-L. Bredas, Adv. Mater. 13 (2001) 1053-1067.

[15] J. Gierschner and S.Y. Park, J. Mater. Chem. C, 1 (2013) 5818-5832.

[16] J. Gierschner, Y.-S. Huang, B. Van Averbeke, J. Cornil, R.H. Friend and D. Beljonne, J. Chem. Phys. 130 (2009) 044105.

[17] Y.X. Xu, H.Y. Zhang, F. Li, F.Z. Shen, H. Wang, X.J. Li, Y. Yu, Y.G. Ma, J. Mater. Chem. 22 (2012) $1592 \mathrm{e} 1597$.

[18] J. Deng, J. Tang, Y. Xu, L. Liu, Y. Wang, Z. Xie and Y. Ma, Phys. Chem. Chem. Phys. 17 (2015) 3421-3425.

[19] H. Wang, F. Sun , Y. Zhao, P. Lu, B. Wang, H. Wang, Organic Electronics 28 (2016) 287-293.

[20] G. Fan, D. Yan, Scientific Reports 4 (2014) 4933.

[21] Y. Li, F. Li, H. Zhang, Z. Xie, W. Xie, H. Xu, B. Li, F. Shen, L. Ye, M. Hanif, D. Ma, Y. Ma, Chem. Commun. (2007) 231-233. 
[22] J. Deng, Y. Xu, L. Liu, C. Feng, J. Tang, Y. Gao, Y. Wang, B. Yang, P. Lu, W. Yang and Y. Ma, Chem. Commun. (2016) DOI: 10.1039/c5cc09702a.

[23] K. Bando, T. Nakamura, S. Fujiwara, Y. Masumoto, F. Sasaki, S. Kobayashi, Y. Shimoi, S. Hotta, Phys. Rev. B. 77 (2008) 045205.

[24] S. Varghese, S.-J. Yoon, E.M. Calzado, S. Casado, P.G. Boj, M.A. Diaz-Garcia, R. Resel, R. Fischer, B. Mili’an-Medina, R. Wannemacher, S. Y. Park and J. Gierschner, Adv. Mater. 24 (2012) 6473-6478.

[25] Gaussian, Inc., Wallingford CT, 2009.

[26] S.J. Yoon, J.W. Chung, J. Gierschner, K.S. Kim, M.G. Choi, D. Kim, S.Y. Park, J. Am. Chem. Soc. 132 (2010) 13675-13683.

[27] S.-J. Yoon, S. Varghese, S. K. Park, R. Wannemacher, J. Gierschner and S. Y. Park, Adv. Opt. Mater. 1 (2013) 232-237. 

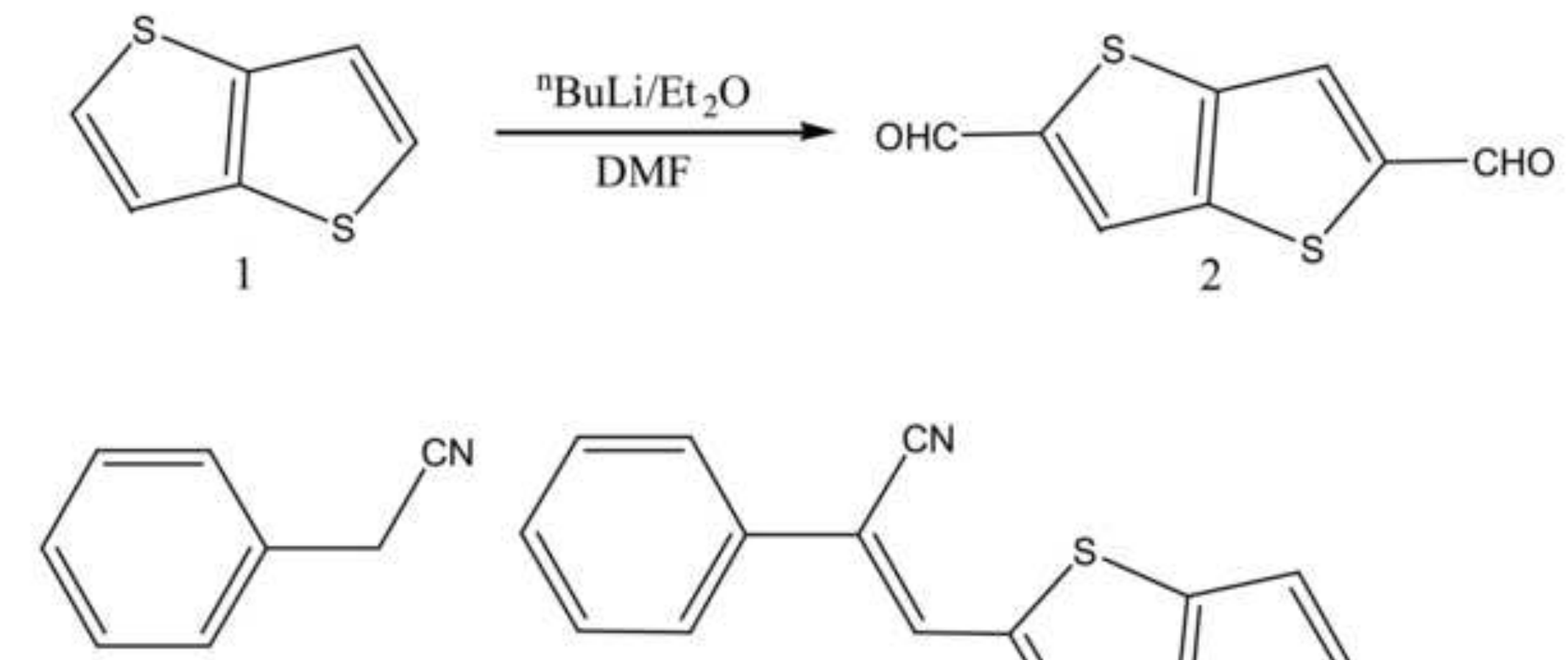

$\underset{\mathrm{THF} / \mathrm{t}-\mathrm{BuOH}}{\stackrel{\mathrm{TBAH} / \mathrm{t}-\mathrm{BuOK}}{\longrightarrow}}$

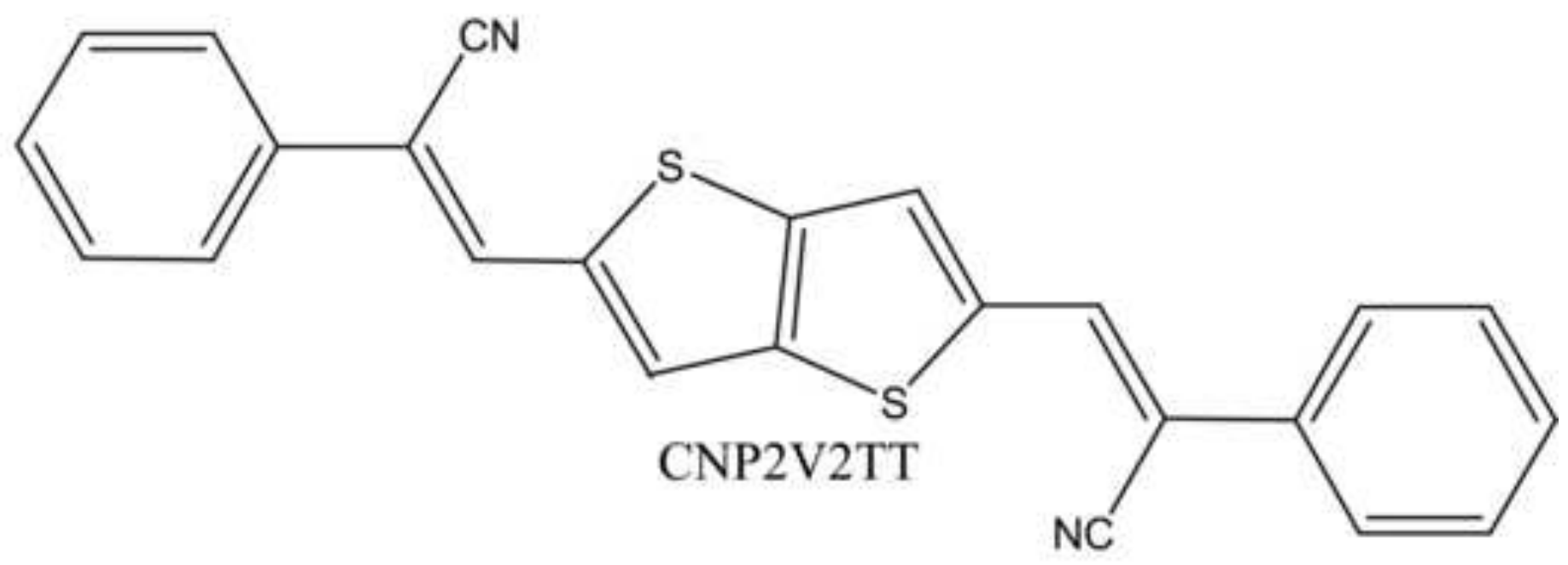




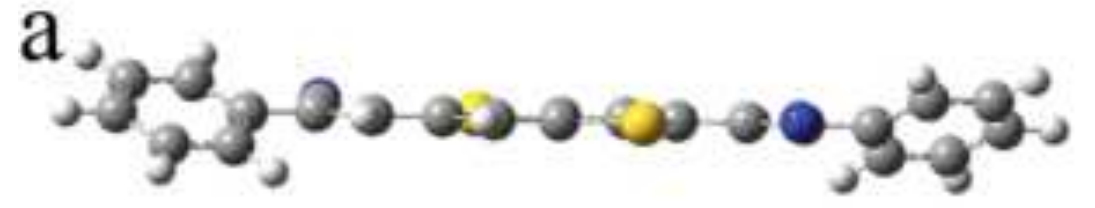

Twisted conformation

$$
\text { PL yield }<1 \%
$$

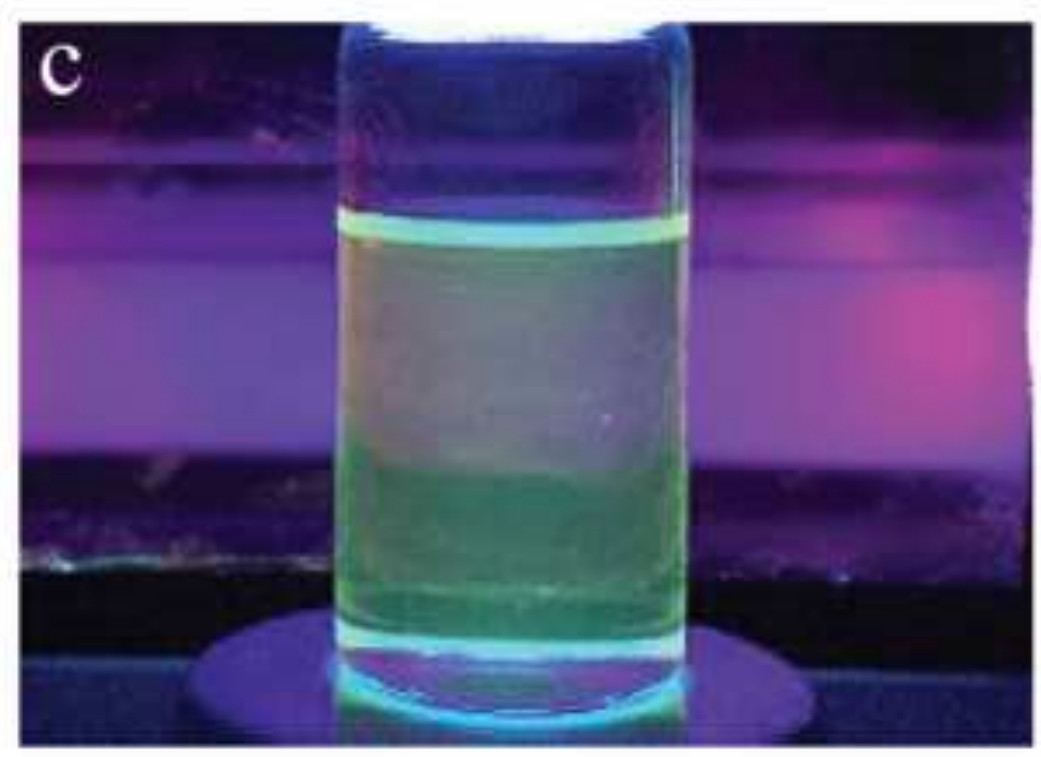

b

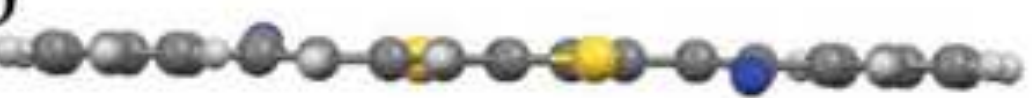

Coplanar conformation PL yield up to $37 \%$

AIE

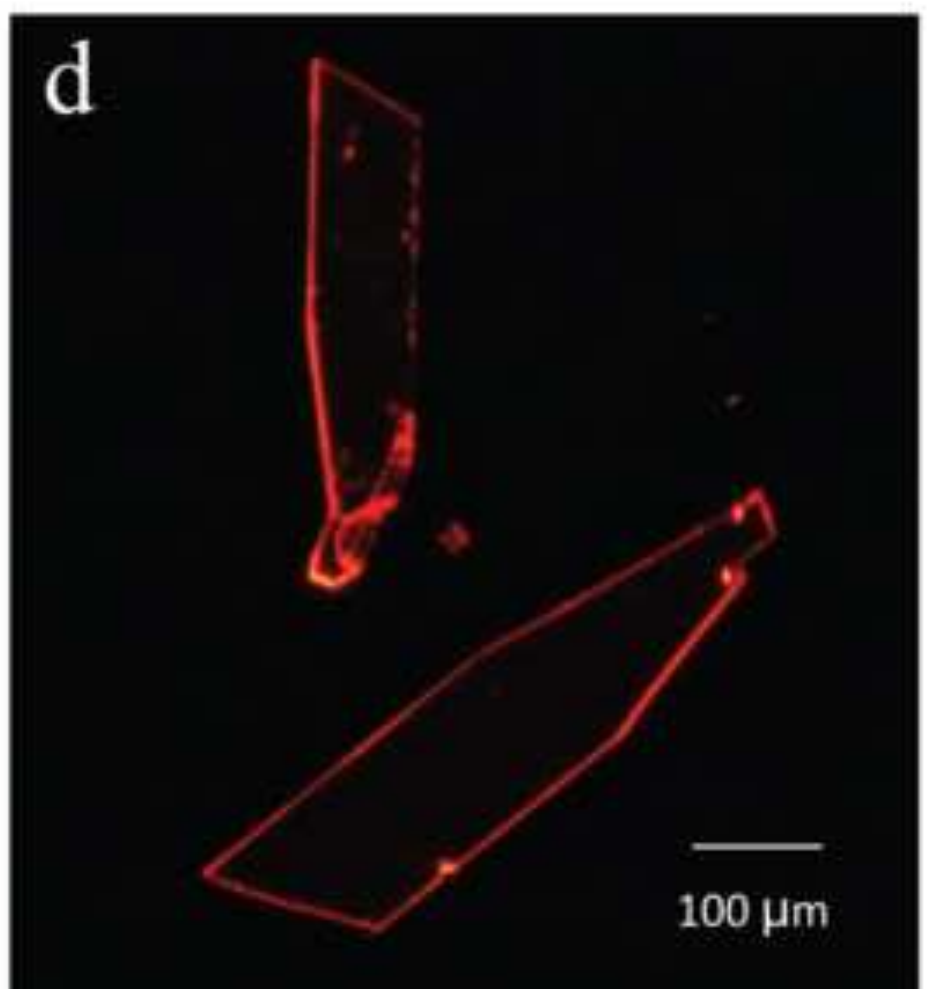




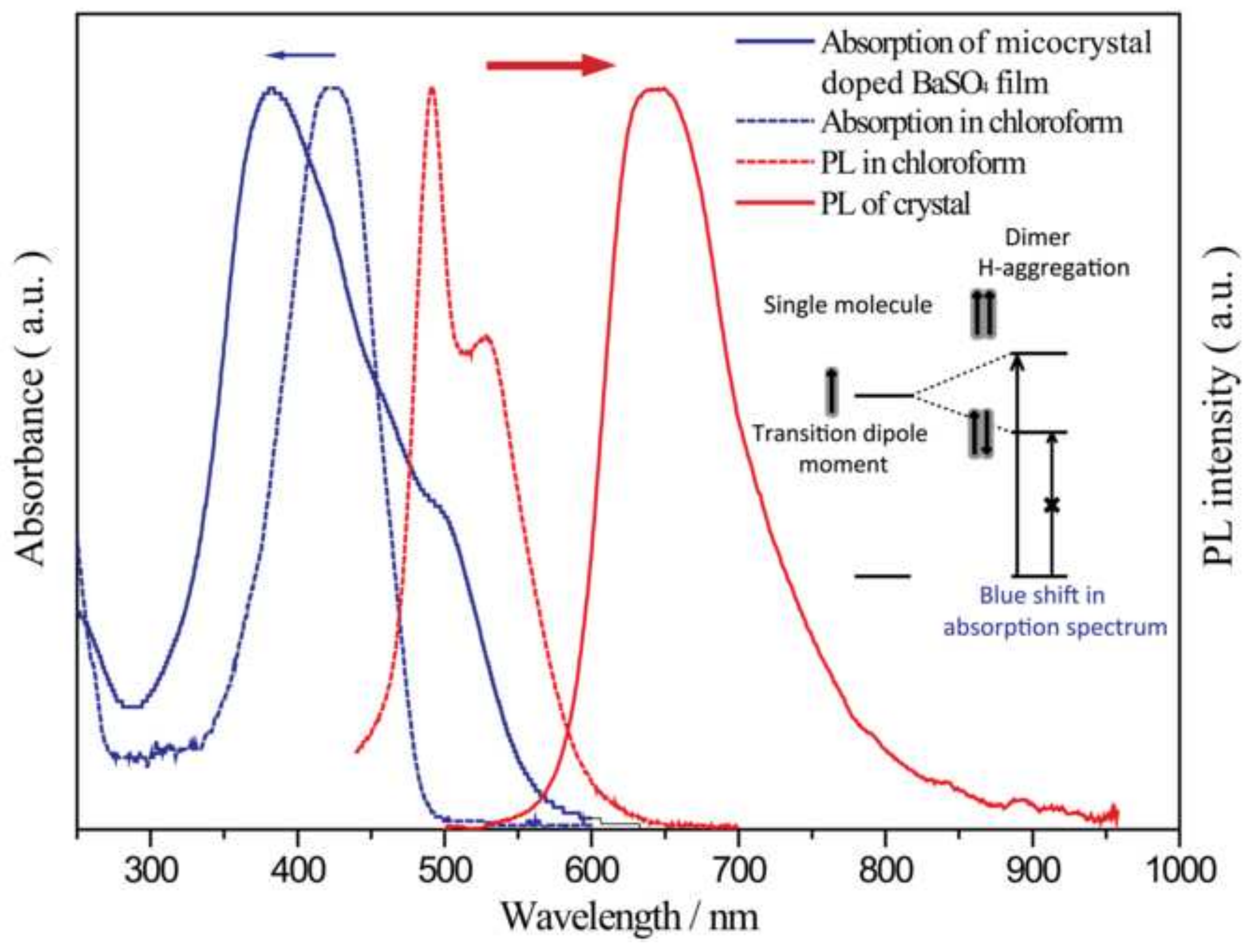



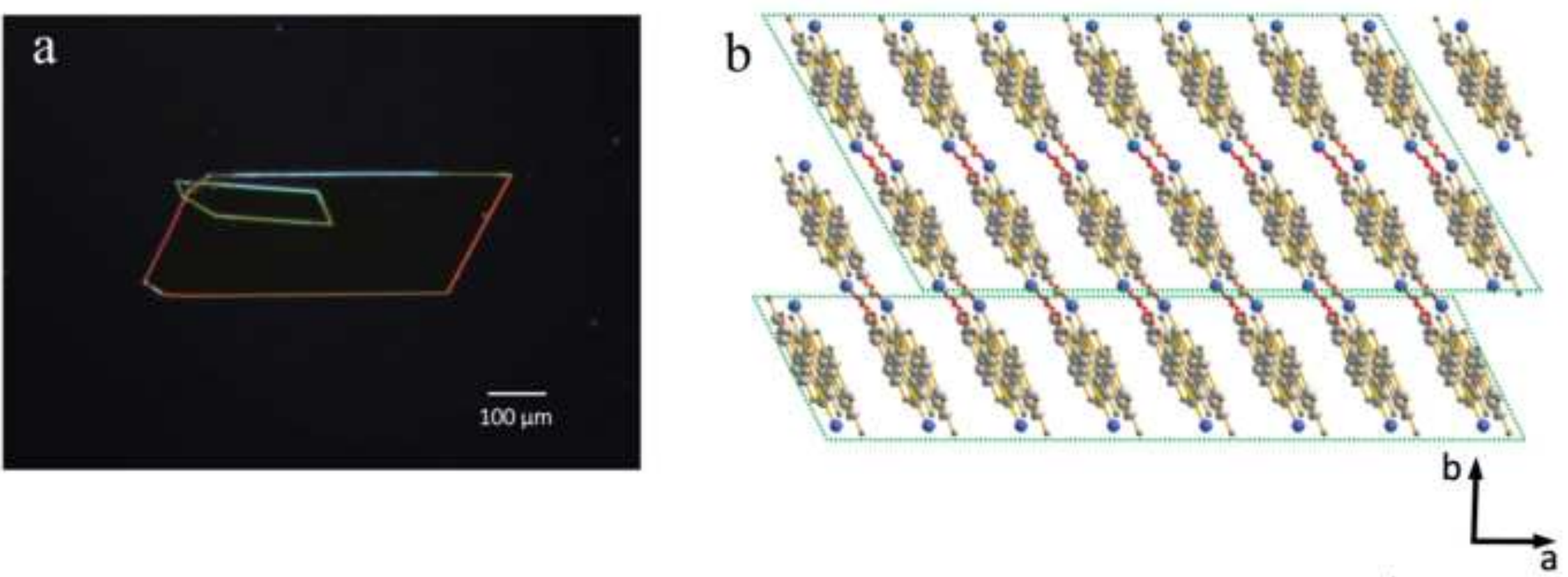

normal

C

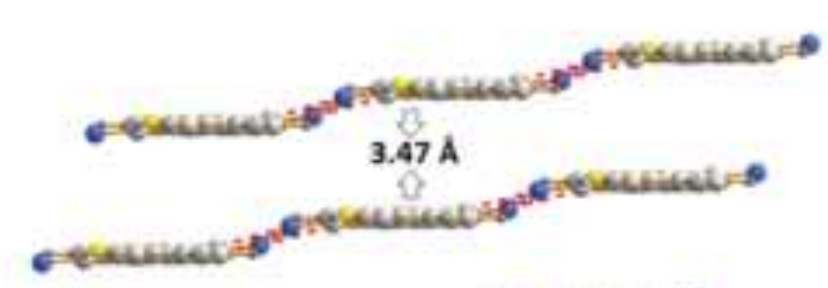

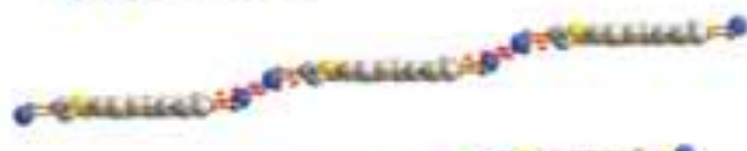

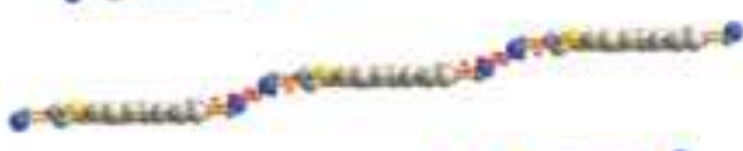

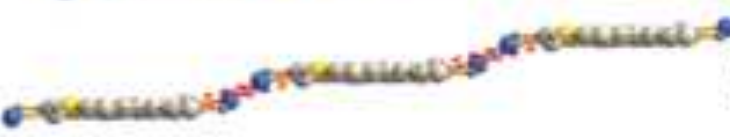
${ }_{c}^{b} y^{a}$

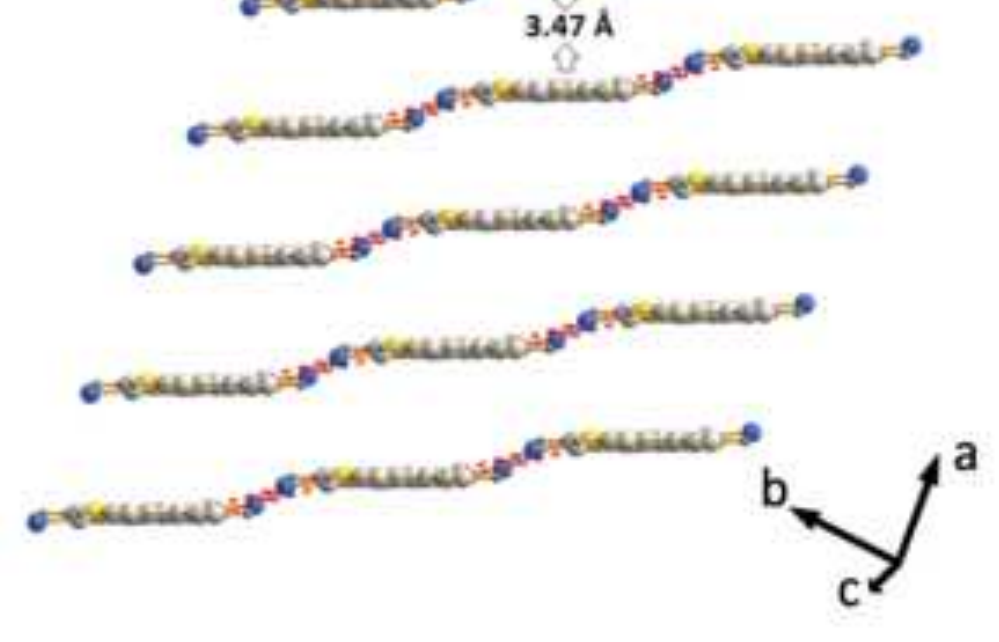

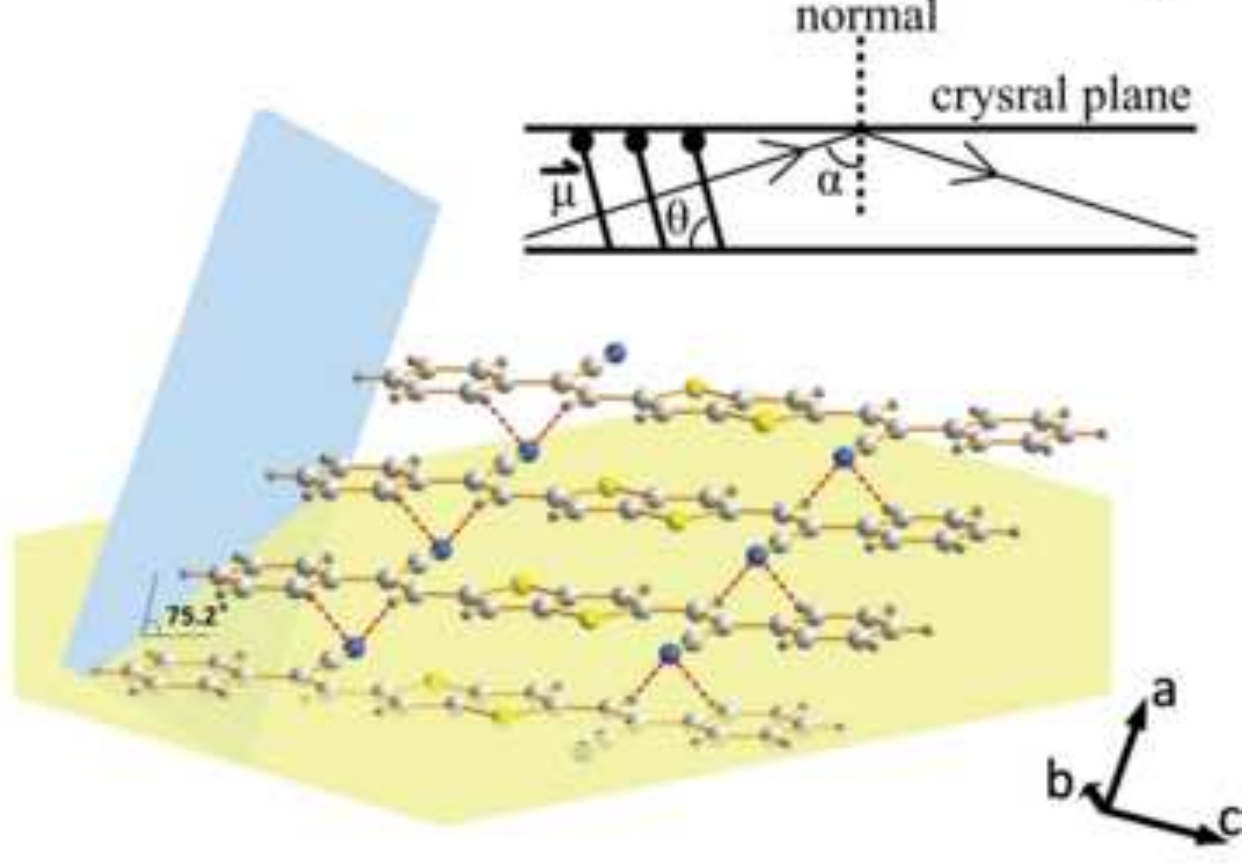

d 

a номо द्वें

b

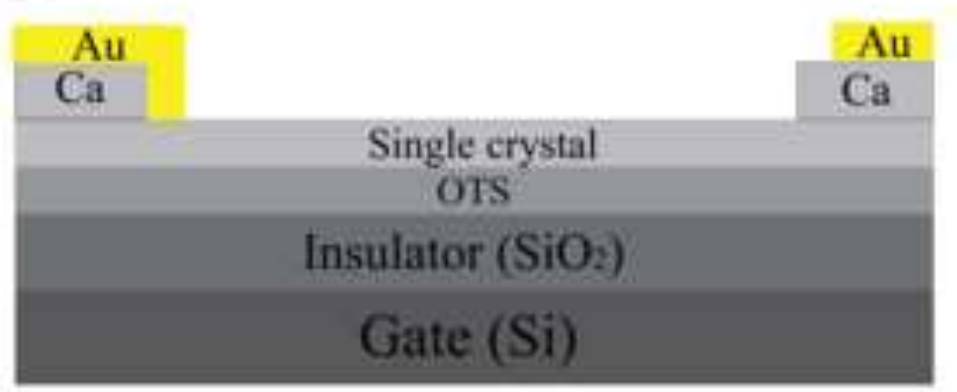

c
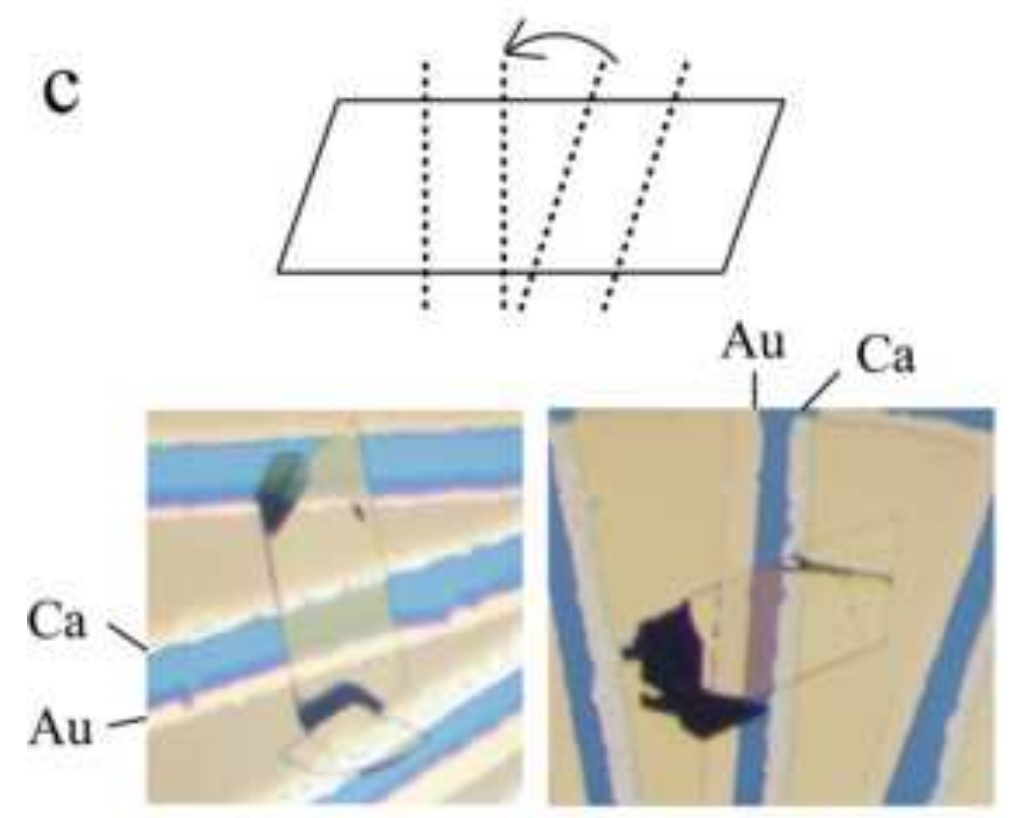
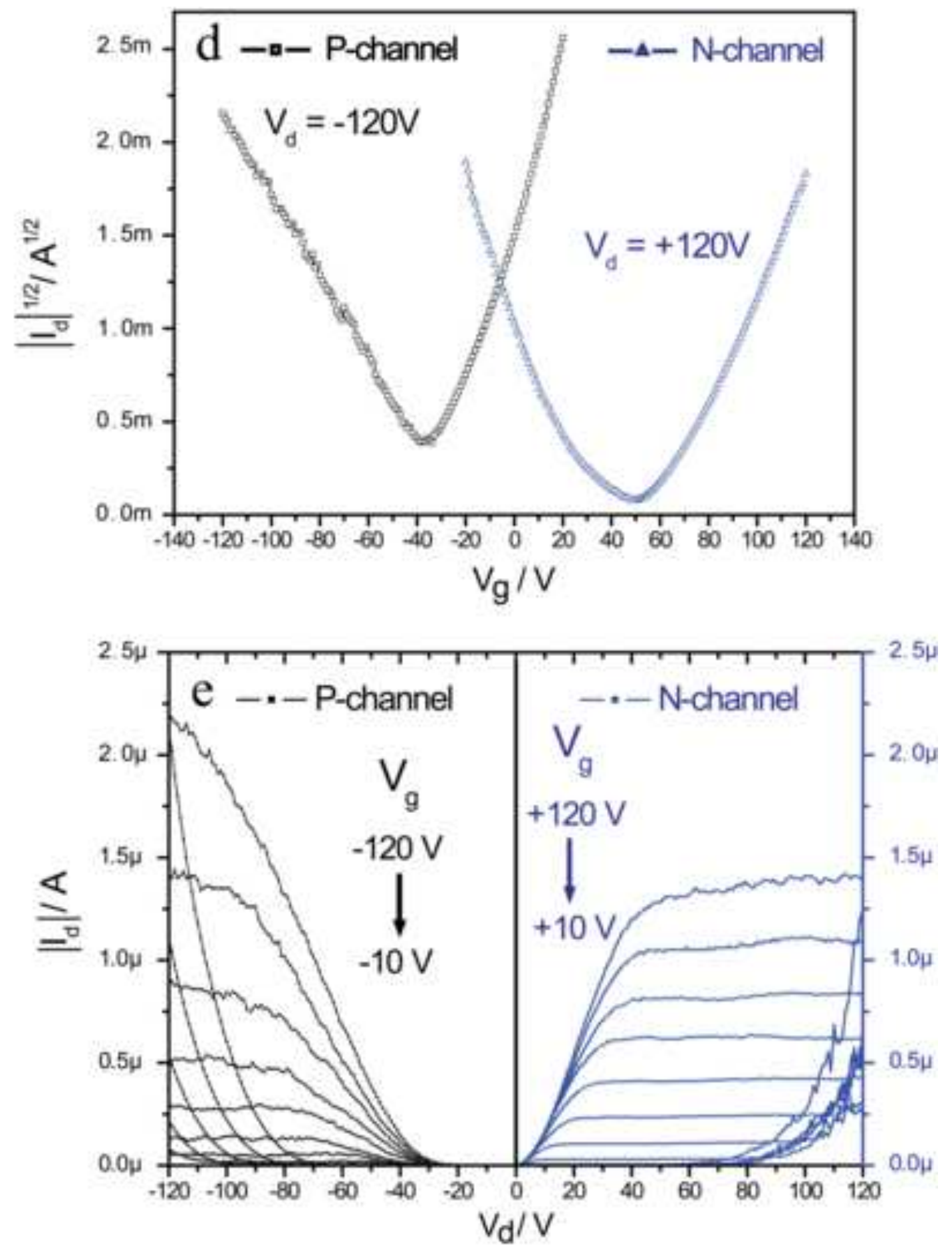